\title{
Risk-Constrained Self-Scheduling of a Thermal Power Producer
}

\author{
Antonio J. Conejo, Fellow, IEEE, Francisco J. Nogales, José M. Arroyo, Member, IEEE, and \\ Raquel García-Bertrand, Student Member, IEEE
}

\begin{abstract}
This paper addresses the self-scheduling problem of a price-taker power producer. It focuses on risk modeling, emphasizing the tradeoff existing between maximum profit and minimum risk. The paper analyzes a self-scheduling model that considers simultaneously profit and risk. This model is formulated as a mixed-integer quadratic programming problem, which is solved using commercially available software. Relevant results from a realistic case study are discussed.
\end{abstract}

Index Terms-Pool-based electricity market, price-taker power producer, profit versus risk tradeoff, risk-constrained self-scheduling.

\section{NOMENCLATURE}

\section{Variables:}

$c_{t} \quad$ Production cost during hour $t$.

$p_{t} \quad$ Power production during hour $t$

$R \quad$ Total revenue.

$V \quad T \times T$ covariance matrix of random variables $\lambda_{1}, \ldots, \lambda_{T}$.

$\lambda_{t} \quad$ Market-clearing price of hour $t$ (random variable).

$\Lambda_{d} \quad$ Vector of the $T(24)$ prices for day $d$. Constants:

$D \quad$ Number of days for which true and estimate prices are available.

$T \quad$ Considered time periods in one day (typically 24).

$\alpha \quad$ Factor used to estimate the covariance matrix.

$\beta \quad$ Weighting factor to incorporate risk into the expected profit objective function.

$\Pi \quad$ Feasible operating region of the generating machine. Miscellaneous:

$E_{\lambda_{1}, \ldots, \lambda_{T}}$ Expected value operator with respect to random variables $\lambda_{1}, \ldots, \lambda_{T}$.

$\operatorname{Var}_{\lambda_{1}, \ldots, \lambda_{T}}$ Variance operator with respect to random variables $\lambda_{1}, \ldots, \lambda_{T}$.

est Superscript that indicates estimate value.

true Superscript that indicates true value.

exp Superscript that indicates expected value.

Manuscript received February 10, 2004. The work of A. J. Conejo, J. M. Arroyo, and R. García-Bertrand was supported in part by the Ministry of Science and Technology of Spain through the CICYT Project DPI2003-01362 and by Junta de Comunidades de Castilla-La Mancha through Project GC-02-006.

A. J. Conejo, J. M. Arroyo, and R. García-Bertrand are with the Department of Electrical Engineering, University of Castilla-La Mancha, Ciudad Real, Spain (e-mail: Antonio.Conejo@uclm.es; JoseManuel.Arroyo@uclm.es; Raquel.Garcia@uclm.es).

F. J. Nogales is with the Department of Statistics, Universidad Carlos III, Madrid, Spain (e-mail: FcoJavier.Nogales@uc3m.es).

Digital Object Identifier 10.1109/TPWRS.2004.831652

\section{INTRODUCTION}

$\mathbf{T}$ HIS PAPER considers a day-ahead electric energy market based on a pool. Within this pool, producers and consumers submit production and consumption bids to the market operator, which clears the market using an appropriate market-clearing procedure. This procedure results in 24 hourly energy prices to be paid by consumers and to be charged by producers. Appropriate forecasting techniques can be used to predict these 24 day-ahead hourly prices. Furthermore, the covariance matrix that expresses the statistical dependence of these prices among themselves can also be estimated [1]. It should be noted that modeling bilateral contracts requires longer market horizons than those considered in this paper.

In the above framework, this paper addresses the self-scheduling problem faced by a power producer. The effect of risk is explicitly recognized in formulating this self-scheduling problem taking into account the variance of the market-clearing prices. Therefore, the tradeoff of maximum profit versus minimum risk is properly addressed. Within the framework stated in [2], the analysis performed can be extended to simultaneously consider AGC, spinning reserve, and nonspinning reserve markets.

Note that any producer participating in an electric energy pool should self-schedule its units to maximize its expected profit assuming a given level of risk. This optimal self-schedule is then used by the producer to derive an appropriate bidding strategy to the pool [3]. This strategy is designed so that the optimal selfschedule is accepted by the pool operator through its marketclearing procedure (based on unit commitment or on auctions).

The producer considered in this paper is a price-taker, i.e., a producer with no capability of altering the market-clearing prices. Therefore, its power plants do not have to seek coordination among themselves in determining their respective production strategies because coordination does not change marketclearing prices. Therefore, the self-scheduling of each generator is independent of the self-scheduling of the others. For the sake of simplicity and without loss of generality, a producer owning a single generator is considered in this paper.

The objective of the producer is to maximize the expected value of profit from selling energy in the day-ahead market, assuming a certain risk level. Therefore, a precise modeling of risk is embedded in the considered maximum profit problem.

The profit maximization problem faced by the producer is therefore a risk-constrained self-scheduling problem that is formulated as a mixed-integer quadratic programming problem. A commercial software [4] is used to solve this problem.

This paper is built upon previous results on self-scheduling of power producers as stated in [3] and [5]. Its relevant contribution 
consists in modeling risk in a rigorous manner and providing a tradeoff decision framework involving profit versus risk.

Relevant references on self-scheduling include [5] and [6]. Although several references are available on risk management in pool markets for a long-term time span, only few references address risk-related problems in the short-term. They include the following references. Paper [7] presents a unit commitment problem with uncertainty in the market prices. It uses a real option model to manage the price risk and the price behavior is modeled through scenarios (trinomial trees). Paper [8] presents "Value-at-Risk" and hedging instruments to manage market price risk for suppliers, distributors and traders. In [9], the authors analyze how to deal with the risk associated with operating conditions for models that include the transmission network. In [10], the authors explain how the participants in the former California power market had a risk exposure related to prices and propose the "Value-at-Risk" method to quantify this exposure. [11] presents a methodology to analyze the risk associated to short-term operational planning in the presence of load uncertainty.

The risk analysis carried out in this paper frames itself in a pool-based electric energy market that is cleared using a simple auction procedure, and where bilateral contracts are not significant. This is actually the case of the electricity market of mainland Spain [12].

If the producer has the possibility of signing long/medium-term contracts to hedge risk, the decision framework becomes more complex and versatile. In the long/medium-term, the producer should decide which contracts to sign, and as a result of such decision, the power that the producer allocates to trade in the pool (short-term). The long/medium-term decision of which contracts to sign is better taken within a stochastic programming framework, similar to the one stated, for instance, in [13]-[16] or [17]. The power that has not been allocated to bilateral contracts should be traded in the spot market using a framework similar to the one developed in this paper. An overview of the cascaded decisions faced by a power producer in the long-, medium-, and short-term frameworks can be found in [18].

This paper is organized as follows. Section II provides a description of the revenue that the producer expects to achieve in the day-ahead energy market. This revenue is characterized as a random variable whose average value and variance (a measure of risk) are described mathematically. In Section III, the riskconstrained self-scheduling problem faced by the producer is formulated as a mixed-integer quadratic programming problem. Results from a realistic case study are described and analyzed in Section IV. Finally, Section V provides some relevant conclusions.

\section{REVENUE AND RISK CHARACTERIZATION}

The expected value of the revenue obtained by the generator in the day-ahead electric energy market is calculated as

$$
R^{\exp }=\mathrm{E}_{\lambda_{1}, \ldots, \lambda_{T}}\left\{\sum_{t=1}^{T} \lambda_{t} p_{t}\right\}
$$

Note that expectation and summation operators can be swapped, i.e.,

$$
R^{\exp }=\sum_{t=1}^{T} \mathrm{E}_{\lambda_{t}}\left\{\lambda_{t}\right\} p_{t}=\sum_{t=1}^{T} \lambda_{t}^{\mathrm{est}} p_{t}
$$

Therefore, the average value of the revenue of the generator in the day-ahead electric energy market is computed as the summation over time of the price estimate times the actual power production in each hourly time-period.

The mutual dependence among the 24 revenues can be measured through their variance. This variance is an appropriate measure of risk: the higher the variance, the higher the risk. The variance of the 24 revenues can be computed as

$$
\operatorname{Var}_{\lambda_{1}, \ldots, \lambda_{T}}\left\{\sum_{t=1}^{T} \lambda_{t} p_{t}\right\}=\sum_{i=1}^{T} \sum_{j=1}^{T} V_{i j} p_{i} p_{j}
$$

where $V=\left(V_{i j}\right)$ is the $T \times T$ covariance matrix of prices $\lambda_{1}, \ldots, \lambda_{T}$ [1]. It should be noted that the variance of the total revenue can be formulated solely as a function of the covariance matrix of the prices because the only random variables involved are these prices.

The actual covariance matrix $V$ for day $d$ is

$$
V=\mathrm{E}_{\lambda_{1}, \ldots, \lambda_{T}}\left\{\left(\Lambda_{d}^{\text {true }}-\Lambda_{d}^{\mathrm{est}}\right)\left(\Lambda_{d}^{\text {true }}-\Lambda_{d}^{\mathrm{est}}\right)^{\mathrm{T}}\right\}
$$

where $\Lambda_{d}=\left[\lambda_{1}, \ldots, \lambda_{T}\right]^{\mathrm{T}}$ for day $d$.

If the true values of prices as well as their estimates are available up to day $d-1$, the covariance matrix of day $d$ can be estimated as

$$
V^{\text {est }}=\frac{1}{D} \sum_{i=1}^{D}\left(\Lambda_{i}^{\text {true }}-\Lambda_{i}^{\text {est }}\right)\left(\Lambda_{i}^{\text {true }}-\Lambda_{i}^{\text {est }}\right)^{\mathrm{T}}
$$

where $D$ is a convenient number of days (up to and including day $d-1$ ) for which true and estimate prices are available.

Hourly price series of most electric energy markets present some characteristics that can cause problems if (5) is directly used. These characteristics are [19]: nonconstant mean and variance, multiple seasonality (corresponding to a daily and weekly periodicity, respectively), high volatility, and high percentage of unusual prices (mainly in periods of high demand). Therefore, a better estimate for the covariance matrix can be obtained using the following exponentially weighted moving-average equation [20]:

$$
\begin{array}{r}
V^{\mathrm{est}}=(1-\alpha) \sum_{i=1}^{D} \alpha^{i-1}\left(\Lambda_{D-i+1}^{\text {true }}-\Lambda_{D-i+1}^{\mathrm{est}}\right) \\
\quad \times\left(\Lambda_{D-i+1}^{\text {true }}-\Lambda_{D-i+1}^{\mathrm{est}}\right)^{\mathrm{T}}
\end{array}
$$

where $D$ is greater than or equal to 24 to make the covariance matrix positive definite. To guarantee that covariance matrix $V$ is positive definite, at least 24 error vectors should be linearly 
independent, which is usually the case. Moreover, using more than 24 error vectors further ensures the positive definiteness of the covariance matrix $V$. Because past prices are weighted by the "smoothing constant" $\alpha(0<\alpha<1)$, higher weights are assigned to the days closer to day $d$ and these weights decay exponentially as the days considered are farther and farther away in the past from day $d$. Therefore, seasonality effects and outliers have less impact on variances and covariances as these effects occur in periods more and more distant from day $d$. It should be noted that both (5) and (6) render biased estimations of matrix $V$. However, as stated in [20], it can be shown that the bias approaches zero as $D$, the number of days considered in the estimation, increases. Nevertheless, as the value of $D$ increases, precision may deteriorate due to possible outliers. Therefore, by trial and error, an appropriate value of $D$ should be selected so that the bias approaches zero while estimation precision remains high.

\section{SELF-SCHEDULING}

At the time of self-scheduling, any producer faces a tradeoff between maximum profit and minimum risk. If the producer decides to ignore risk, the resulting profit maximization selfscheduling problem can be formulated as [5]

$$
\begin{aligned}
& \underset{p_{1}, \ldots, p_{T}}{\operatorname{maximize}} \sum_{t=1}^{T}\left(\lambda_{t}^{\text {est }} p_{t}-c_{t}\right) \\
& \text { subject to } p_{1}, \ldots, p_{T} \in \Pi
\end{aligned}
$$

where $c_{t}$ is the production cost during hour $t$. This cost includes a quadratic operation cost that depends on the power output $p_{t}$, and fixed, start-up and shut-down costs. Further information on cost can be found in [5].

Operation constraints of the generator include:

- minimum and maximum power output limits;

- ramp-up and ramp-down limits;

- start-up and shut-down ramp limits;

- minimum up- and minimum down-time constraints.

The above constraints can be generally expressed as $p_{1}, \ldots, p_{T} \in \Pi$, where $\Pi$ is the feasible operating region of the generator. A detailed description of the feasibility region $\Pi$ can be found in [5].

On the other hand, if the producer seeks to minimize risk and it is not disturbed by low profit, the corresponding risk minimization self-scheduling problem can be formulated as

$$
\begin{aligned}
& \underset{p_{1}, \ldots, p_{T}}{\operatorname{minimize}} \sum_{i=1}^{T} \sum_{j=1}^{T} V_{i j}^{\mathrm{est}} p_{i} p_{j} \\
& \text { subject to } p_{1}, \ldots, p_{T} \in \Pi
\end{aligned}
$$

where both $i$ and $j$ are time indices.

It should be noted that problem (8) is of no practical use. However, it is formulated for derivation consistency and for the sake of clarification.

Generally, a producer is interested in finding a self-schedule that results in a large profit with low risk (variance). To combine
TABLE I

TECHNICAL DATA FOR THE GENERATING MACHINE

\begin{tabular}{cccc}
\hline $\begin{array}{c}\text { Minimum } \\
\text { power [MW] }\end{array}$ & $\begin{array}{c}\text { Maximum } \\
\text { power [MW] }\end{array}$ & $\begin{array}{c}\text { Start-up ramping } \\
\text { limit [MW/h] }\end{array}$ & $\begin{array}{c}\text { Shut-down ramping } \\
\text { limit [MW/h] }\end{array}$ \\
\hline 112 & 294 & 170 & 160 \\
\hline $\begin{array}{c}\text { Ramping-up } \\
\text { limit [MW/h] }\end{array}$ & $\begin{array}{c}\text { Ramping-down } \\
\text { limit [MW/h] }\end{array}$ & $\begin{array}{c}\text { Minimum } \\
\text { up-time [hours] }\end{array}$ & $\begin{array}{c}\text { Minimum } \\
\text { down-time [hours] }\end{array}$ \\
\hline 60 & 50 & 4 & 4 \\
\hline
\end{tabular}

TABLE II

COSTS DATA For THE GENERATING MACHINE

\begin{tabular}{ccccc}
\hline $\begin{array}{c}\text { Fixed } \\
{[\$ / \mathrm{h}]}\end{array}$ & $\begin{array}{c}\text { Linear } \\
{[\$ / \mathrm{MWh}]}\end{array}$ & $\begin{array}{c}\text { Quadratic } \\
{\left[\$ / \mathrm{MW}^{2} \mathrm{~h}\right]}\end{array}$ & $\begin{array}{c}\text { Start-up } \\
{[\$ / \text { start-up }]}\end{array}$ & $\begin{array}{c}\text { Shut-down } \\
{[\$ / \text { shut-down }]}\end{array}$ \\
\hline 1150 & 18 & 0.035 & 1038 & 56 \\
\hline
\end{tabular}

TABLE III

PRICE ESTIMATES

\begin{tabular}{cc|cc}
\hline Hour \# & $\begin{array}{c}\text { Price estimate } \\
{[\$ / \mathrm{MWh}]}\end{array}$ & Hour $\#$ & $\begin{array}{c}\text { Price estimate } \\
{[\$ / \mathrm{MWh}]}\end{array}$ \\
\hline 1 & 33.31 & 13 & 41.05 \\
\hline 2 & 26.53 & 14 & 41.61 \\
\hline 3 & 22.16 & 15 & 38.98 \\
\hline 4 & 23.10 & 16 & 39.74 \\
\hline 5 & 22.60 & 17 & 42.02 \\
\hline 6 & 23.15 & 18 & 42.09 \\
\hline 7 & 24.65 & 19 & 40.74 \\
\hline 8 & 24.75 & 20 & 38.80 \\
\hline 9 & 25.50 & 21 & 39.63 \\
\hline 10 & 27.58 & 22 & 46.14 \\
\hline 11 & 31.60 & 23 & 39.04 \\
\hline 12 & 35.60 & 24 & 33.68 \\
\hline
\end{tabular}

these two conflicting objectives, the technique presented in [21] for portfolio selection can be used. This technique uses a single objective function with the help of a risk tolerance parameter $\beta$. The resulting self-scheduling problem has the form

$$
\begin{aligned}
& \underset{p_{1}, \ldots, p_{T}}{\operatorname{maximize}} \sum_{t=1}^{T}\left(\lambda_{t}^{\text {est }} p_{t}-c_{t}\right)-\beta \sum_{i=1}^{T} \sum_{j=1}^{T} V_{i j}^{\text {est }} p_{i} p_{j} \\
& \text { subject to } p_{1}, \ldots, p_{T} \in \Pi .
\end{aligned}
$$

Note that the variance of the total revenue is equal to the variance of the total profit because all costs are considered deterministic.

Penalty parameter $\beta$ allows adding the two conflicting terms that form the objective function of problem (9). Parameter $\beta$ lies in the range $[0, \infty)$ and its actual value materializes the tradeoff between expected profit and risk; therefore, it depends on the preferences of the producer. A conservative producer places more emphasis on minimizing risk while deriving its self-scheduling, so it chooses a large value of $\beta$ to increase the weight of the risk measure in (9). On the contrary, another producer may be prepared to assume higher risk in the hope of obtaining a higher profit, so its selected value for $\beta$ is close to 0 .

The solution of (9) for different values of $\beta$ provides the efficient frontier [21]-[24], that is, the set of solutions for which expected profit cannot be increased without increasing profit variance, i.e., risk. As stated in [25], the selection of the weighting factor $\beta$ depends on, among others, the financial situation of the 
TABLE IV

ESTIMATE OF THE COVARIANCE MATRIX

\begin{tabular}{|c|c|c|c|c|c|c|c|c|c|c|c|c|c|c|c|c|c|c|c|c|c|c|c|c|}
\hline $\begin{array}{c}\text { Hour } \\
\#\end{array}$ & 1 & 2 & 3 & 4 & 5 & 6 & 7 & 8 & 9 & 10 & 1 & 12 & 13 & 4 & 15 & 16 & 17 & 18 & 19 & م2 & 21 & 22 & 23 & 24 \\
\hline 1 & 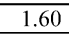 & 40 & & & & 000 & 010 & 058 & & & & & & & 014 & & & & & & & & & 80 \\
\hline 2 & 10 & & & & & & & & & & & & & & & & & & & & & & & \\
\hline 3 & & & & & & & & 127 & & & & & & & & & & & & 0.00 & & & & -0.10 \\
\hline 4 & & 0.00 & & & & & & & & 1 & & & & & & & & & & 0.00 & & & & -0.05 \\
\hline 5 & & & & & 025 & & & & 0 & & & & & & & & & & & 0.01 & & & & \\
\hline 6 & & & & & & & & & & & & & & & & & & & & & & & & \\
\hline 7 & & & & & & & & & & & & & & & & & & & & -0 & & & & -0.20 \\
\hline 8 & & & & & & & & & & & & & & & & & & & & & & & & \\
\hline 9 & & & & & & & & & & & & & & & & & & & & & & & & -0.25 \\
\hline 10 & & 8 & & & & & & & & & & & & & & & & & & & & & & -0.15 \\
\hline 11 & 09 & -0.12 & 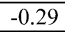 & 7 & 1 & 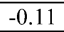 & 0 & 0.01 & & & & & & & & & & & & & & & & 0.01 \\
\hline 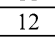 & & & & & & & & & & & & & & & & & & & & & & & & \\
\hline 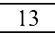 & & & & & & & & & & & & & & & & & & & & & & & & \\
\hline 14 & & -0.0 & & & & & & & & & & & & & & & & & & & & & & 0.17 \\
\hline 15 & 17 & 0.0 & & 9 & & -0 & 0 & -0.38 & 9 & 0 & & . & & & & & & & & 0 & & & 40 & -0.05 \\
\hline 16 & & & & & & & & & & & & & & & & & & & & 0.08 & & & -0.08 & -0.04 \\
\hline 17 & & 0.0 & 0.29 & 0.1 & & & & 0.39 & & & & & & & & & & & & 0 . & & & 0.05 & -0.22 \\
\hline 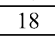 & & & & & & & & & & & & & & & & & & & & & & & & \\
\hline & & & & & & & & & & & & & & & & & & & & & & & & \\
\hline 20 & & & & & & & & & & & & & & & & & & & & & & & & \\
\hline 21 & & -0.20 & & .04 & & .1 & -0.65 & -0.56 & -0.39 & & & -0.07 & & & & & -0 . & -0. & & 0.03 & 0.66 & & .56 & -0.02 \\
\hline 22 & & & & .02 & & & & & & & & & & & & & & & & & & & 45 & 0.26 \\
\hline 23 & -0.19 & -0.15 & & 0.06 & & - & 1 & -0.59 & -0 . & -0 & & -0 & & & & -0 & 0. & -0 & & 0.23 & 0.56 & .45 & 0.84 & -0.03 \\
\hline 24 & -0.01 & 0.10 & -0.10 & -0.05 & -0.15 & -0.12 & -0.26 & -0.44 & -0.25 & -0.15 & 0.01 & 0.20 & -0.15 & 0.17 & -0.05 & -0.04 & -0.22 & 0.07 & -0.01 & 0.09 & -0.02 & 0.26 & -0.03 & 0.60 \\
\hline
\end{tabular}

generating company, its willingness to take risks and the characteristics of the particular electricity market. A detailed discussion on how to obtain appropriate values for the weighting factor $\beta$ is outside the scope of this paper.

It should be noted that modeling the feasible operating region of the generator (represented by $\Pi$ ) requires the use of binary variables to represent the commitment status as well as start-ups and shut-downs. Therefore, problem (9) is a mixed-integer quadratic programming problem.

If covariance matrix $V$ is positive definite, problem (9) has a strict optimum that can be efficiently attained. If, on the other hand, $V$ is positive semidefinite (only other possibility), nonstrict optima may exist and solution algorithms behave not as efficiently as in the positive definite case. Nevertheless, computational experience shows that appropriate solutions are obtained in both cases.

\section{CASE STUdY}

The considered case study consists in the day-ahead selfscheduling of a power producer owning a single generating machine. Data for this machine is provided in Tables I and II.

Price forecasts are provided in Table III while an estimate of the covariance matrix is provided in Table IV. A time series of three months corresponding to the electric energy market of mainland Spain [12] has been used to estimate prices. Price estimates are obtained using a transfer function procedure [19], while the covariance matrix has been estimated through (6) using data for the last 24 days prior to the estimation day. Through numerical simulation it has been verified that good estimations for this covariance matrix are obtained with $\alpha=0.98$ and $D=24$. Using standard clustering techniques [26] to analyze this covariance matrix, the following can be concluded in terms of correlations affecting risk. Hours 20-24 (late-evening peak) are highly correlated among each other and show small correlations with the other hours of the day.

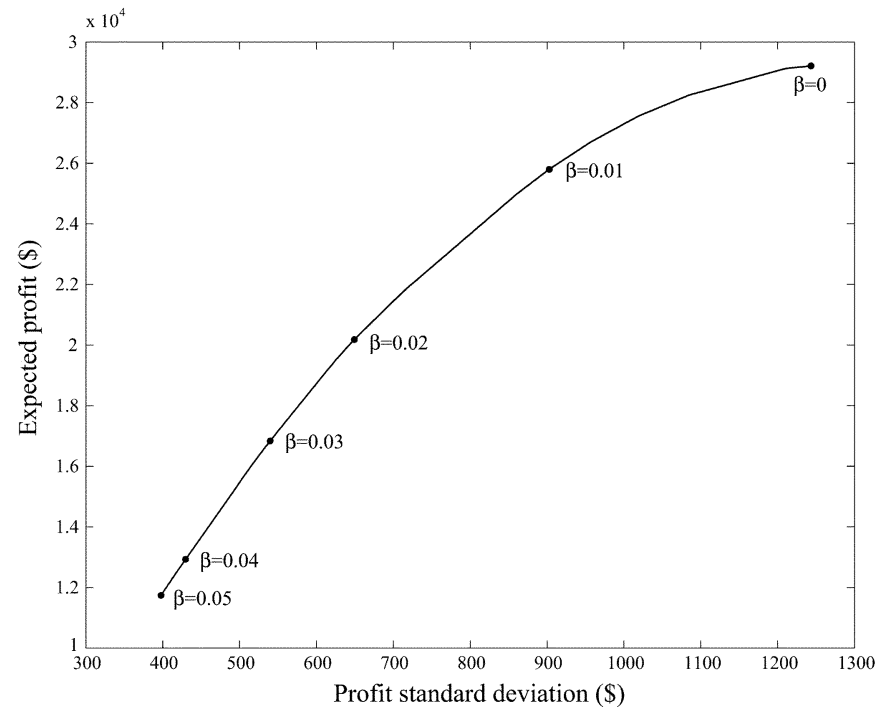

Fig. 1. Expected profit versus profit standard deviation.

The same pattern is presented by hours 5-9 (early-morning shoulder) and 11-14 (afternoon peak). The physical reasons for the above correlations lay on the structure and the composition of the load. However, the study of the load structure is beyond the scope of this paper.

The scheduling problem (9) is solved for different values of the parameter $\beta$, which allows assigning different weights to the risk term versus the profit term in the objective function. This allows constructing the efficient frontier. Fig. 1 depicts expected profit versus profit standard deviation and illustrates the efficient frontier. It can be observed that expected profit increases as variance also increases. Note that the decrement rate in expected profit is important, which makes the tradeoff profit versus risk relevant.

The expected profit achieved by a conservative $\operatorname{producer}(\beta=$ $0.05)$ is $\$ 11737.21$ whereas the expected profit with maximum risk is equal to $\$ 29209.56$. 
TABLE V

SCHEDULING COMPARISON FOR TWO LEVELS OF RISKS

\begin{tabular}{ccc|ccr}
\hline Hour & \multicolumn{2}{c|}{ Power $[\mathrm{MW}]$} & Hour & \multicolumn{2}{c}{ Power $[\mathrm{MW}]$} \\
$\#$ & $\beta=0$ & $\beta=0.05$ & $\#$ & $\beta=0$ & $\beta=0.05$ \\
\hline 1 & 160.00 & 120.00 & 13 & 290.00 & 0.00 \\
\hline 2 & 0.00 & 0.00 & 14 & 294.00 & 0.00 \\
\hline 3 & 0.00 & 0.00 & 15 & 294.00 & 0.00 \\
\hline 4 & 0.00 & 0.00 & 16 & 294.00 & 163.72 \\
\hline 5 & 0.00 & 0.00 & 17 & 294.00 & 172.67 \\
\hline 6 & 0.00 & 0.00 & 18 & 294.00 & 232.67 \\
\hline 7 & 0.00 & 0.00 & 19 & 294.00 & 199.12 \\
\hline 8 & 0.00 & 0.00 & 20 & 294.00 & 150.58 \\
\hline 9 & 0.00 & 0.00 & 21 & 294.00 & 180.34 \\
\hline 10 & 0.00 & 0.00 & 22 & 294.00 & 130.34 \\
\hline 11 & 170.00 & 0.00 & 23 & 287.26 & 0.00 \\
\hline 12 & 230.00 & 0.00 & 24 & 237.26 & 0.00 \\
\hline
\end{tabular}

Table V illustrates scheduling differences for the cases of maximum risk $(\beta=0)$ and a low level of risk $(\beta=0.05)$ (see Fig. 1). This range on $\beta$ is considered wide enough to reflect the variety of risk that electricity producers are willing to assume. As can be seen from this table, the consideration of different risk levels yields two results: 1$)$ the risk averse producer $(\beta=0.05)$ is on-line during fewer time periods, and 2) in those periods in which it is on-line, its production is considerably lower compared to the case of maximum risk $(\beta=0)$. Note that intermediate values of $\beta$ result in different intermediate schedules.

A clear conclusion can be drawn: the producer should make a decision on its desired level of risk before solving its day-ahead scheduling problem and using that information to bid in the electric energy market. Different levels of risk imply different self-scheduling results and different bidding strategies, and ultimately, different actual profits.

The CPU time required to solve problem (9) for a given value of $\beta$ in a Dell PowerEdge 6600 with two processors at $1.60 \mathrm{GHz}$ and $2 \mathrm{~Gb}$ of RAM memory is approximately $7 \mathrm{~s}$.

\section{CONCLUSION}

Any power producer faces a profit versus risk tradeoff while determining its self-scheduling and its bidding strategy for the day-ahead electric energy market. This paper provides an appropriate tool to analyze the profit versus risk tradeoff faced by the producer. Moreover, it provides the producer with an instrument to efficiently self-schedule once a level of risk has been specified. Practical simulations using realistic electric energy markets show that risk levels affect profits in a significant manner. Therefore, any producer should be aware of the consequences of its selected level of risk on its self-scheduling, its bidding strategy, and ultimately, on its actual profits.

\section{REFERENCES}

[1] P. J. Brockwell and R. A. Davis, Time Series: Theory and Methods, 2nd ed. New York: Springer-Verlag, 1991.

[2] J. M. Arroyo and A. J. Conejo, "Optimal response of a power generator to energy, AGC and reserve pool-based markets," IEEE Trans. Power Syst., vol. 17, pp. 404-410, May 2002.

[3] A. J. Conejo, F. J. Nogales, and J. M. Arroyo, "Price-taker bidding strategy under price uncertainty," IEEE Trans. Power Syst., vol. 17, pp. 1081-1088, Nov. 2002.
[4] A. Brooke, D. Kendrick, A. Meeraus, and R. Raman, GAMS: A User's Guide. Washington, DC: GAMS Development Corporation, 1998.

[5] J. M. Arroyo and A. J. Conejo, "Optimal response of a thermal unit to an electricity spot market," IEEE Trans. Power Syst., vol. 15, pp. 1098-1104, Aug. 2000.

[6] S. de la Torre, J. M. Arroyo, A. J. Conejo, and J. Contreras, "Price-maker self-scheduling in a pool-based electricity market: A mixed-integer LP approach," IEEE Trans. Power Syst., vol. 17, pp. 1037-1042, Nov. 2002.

[7] M. Denton, A. Palmer, R. Masiello, and P. Skantze, "Managing market risk in energy," IEEE Trans. Power Syst., vol. 18, pp. 494-502, May 2003.

[8] R. Dahlgren, C. C. Liu, and J. Lawarreé, "Risk assessment in energy trading," IEEE Trans. Power Syst., vol. 18, pp. 503-511, May 2003.

[9] M. Ni, J. D. McCalley, V. Vittal, and T. Tayyib, "Online risk-based security assessment," IEEE Trans. Power Syst., vol. 18, pp. 258-265, Feb. 2003.

[10] R. W. Dahlgren, C. C. Liu, and J. Lawarreé, "Volatility in the California power market: Source, methodology and recommendations," IEE Proc. Gener., Transm., and Distrib., vol. 148, pp. 189-193, Mar. 2001.

[11] A. P. Douglas, A. M. Breipohl, F. M. Lee, and R. Adapa, "Risk due to load forecast uncertainty in short term power system planning," IEEE Trans. Power Syst., vol. 13, pp. 1493-1499, Nov. 1998.

[12] Market Operator of the Electric Energy Market of Mainland Spain. (2002) Tech. Rep. OMEL. [Online]. Available: http://www.omel.es

[13] L. F. Escudero, J. L. de la Fuente, C. García, and F. J. Prieto, "Hydropower generation management under uncertainty via scenario analysis and parallel computation," IEEE Trans. Power Syst., vol. 11, pp. 683-689, May 1996.

[14] M. P. Nowak and W. Römisch, "Lagrangian relaxation applied to power scheduling in a hydro-thermal system under uncertainty," Ann. Oper. Res., vol. 100, pp. 251-272, 2000.

[15] S. Takriti, B. Krasenbrink, and L. S.-Y. Wu, "Incorporating fuel constraints and electricity spot prices into the stochastic unit commitment problem," Oper. Res., vol. 48, pp. 268-280, 2000.

[16] S. E. Fleten, S. W. Wallace, and W. T. Ziemba, "Hedging electricity portfolios via stochastic programming," in Decision-Making Under Uncertainty: Energy and Power, A. Ryszczynski and C. Greengard, Eds. New York: Springer-Verlag, 2002.

[17] N. Growe-Kuska, K. C. Kiwiel, M. P. Nowak, W. Römisch, and I. Wegner, "Power management in a hydro-thermal system under uncertainty by Lagrangian relaxation," in Decision-Making Under Uncertainty: Energy and Power, A. Ryszczynski and C. Greengard, Eds. New York: Springer-Verlag, 2002.

[18] A. J. Conejo and F. J. Prieto, "Mathematical programming and electricity markets," TOP (Sociedad de Estadística e Investigación Operativa), vol. 9, pp. 1-54, 2001.

[19] F. J. Nogales, J. Contreras, A. J. Conejo, and R. Espínola, "Forecasting next-day electricity prices by time series models," IEEE Trans. Power Syst., vol. 17, pp. 342-348, May 2002.

[20] J. P. Morgan, RiskMetrics-Technical Document, 4th ed. New York: Morgan Guaranty Trust Company, 1996.

[21] H. M. Markowitz, "Portfolio selection," J. Finance, vol. 8, pp. 77-91, 1952.

[22] H. Markowitz, Portfolio Selection: Efficient Diversification of Investments. New Haven, CT: Yale Univ. Press, 1959.

[23] E. J. Elton and M. J. Gruber, Modern Portfolio Theory and Investment Analysis, 5th ed. New York: Wiley, 1995.

[24] R. Bjorgan, C. C. Liu, and J. Lawarreé, "Financial risk management in a competitive electricity market," IEEE Trans. Power Syst., vol. 14, pp. 1285-1291, Nov. 1999.

[25] D. G. Luenberger, Investment Science. New York: Oxford Univ. Press, 1997.

[26] A. D. Gordon, Classification. Monographs on Statistics and Probability, 2nd ed. London, U.K.: Chapman \& Hall, 1999.
Antonio J. Conejo (S'86-M'90-SM'98-F'04) received the B.S. degree from the Universidad P. Comillas, Madrid, Spain, in 1983, the M.S. degree from MIT, Cambridge, MA, in 1987, and the Ph.D. degree from the Royal Institute of Technology, Stockholm, Sweden, in 1990.

He is currently a Full Professor at the Universidad de Castilla-La Mancha, Ciudad Real, Spain. His research interests include control, operations and planning and economics of electric energy systems, as well as optimization theory and its applications. 
Francisco J. Nogales received the B.S. degree in mathematics from the Universidad Autónoma de Madrid, Madrid, Spain, in 1995 and the Ph.D. degree in mathematics from the Universidad Carlos III de Madrid in 2000.

$\mathrm{He}$ is currently Assistant Professor in the Department of Statistics at the Universidad Carlos III, Madrid. His research interests include planning and economics of power systems, optimization, and forecasting.

José M. Arroyo (S'96-M'01) received the Ingeniero Industrial degree from the Universidad de Málaga, Málaga, Spain, in 1995, and the Ph.D. degree in power systems operations planning from the Universidad de Castilla-La Mancha, Ciudad Real, Spain, in 2000.

$\mathrm{He}$ is currently an Associate Professor of Electrical Engineering at the Universidad de Castilla-La Mancha. His research interests include operations and planning and economics of electric energy systems, as well as optimization and parallel computation.
Raquel García-Bertrand (S'01) was born in London, U. K., in 1978. She received the Ingeniero Industrial degree from the Universidad de Castilla-La Mancha, Ciudad Real, Spain, in 2001. She is currently pursuing the Ph.D. degree in power systems operations planning at the Universidad de Castilla-La Mancha.

Her research interests include control, operations and planning and economics of electric energy systems. 